\title{
Erratum: Direct measurement of excited-state dipole matrix elements using electromagnetically induced transparency in the hyperfine Paschen-Back regime [Phys. Rev. A 93, 043854 (2016)]
}

\author{
Daniel J. Whiting, James Keaveney, Charles S. Adams, and Ifan G. Hughes
}

(Received 16 March 2018; published 11 April 2018)

DOI: 10.1103/PhysRevA.97.049902

In our paper, on p. $4 \mathrm{Sec}$. IV A we wrote that in a magnetic field of $0.60 \mathrm{~T}$ the states resonantly coupled by the control field can be decomposed in the $\left|m_{L}, m_{S}\right\rangle$ basis as

$$
\begin{gathered}
|g\rangle=0.817\left|0, \frac{1}{2}\right\rangle+0.577\left|1,-\frac{1}{2}\right\rangle, \\
|e\rangle=0.910\left|1, \frac{1}{2}\right\rangle+0.415\left|2,-\frac{1}{2}\right\rangle,
\end{gathered}
$$

and therefore that,

$$
\left|\left\langle g\left|e r_{+1}\right| e\right\rangle\right|=0.982\left|\left\langle 5 P_{3 / 2}, m_{J}=1 / 2\left|e r_{+1}\right| 5 D_{5 / 2}, m_{J}=3 / 2\right\rangle\right| \text {. }
$$

Although it is true that the states can be written in this way, it is irrelevant to the calculation of the dipole matrix element (dme). The correct value of the measured dme is therefore,

$$
\left|\left\langle 5 P_{3 / 2}|| e r|| D_{5 / 2}\right\rangle\right|=\left(2.266 \pm 0.002_{\text {stat }} \pm 0.04_{\text {syst }}\right) e a_{0},
$$

which we note is within the systematic uncertainty of the value originally given. Similarly, the final line of Sec. IV A should be updated to reflect this change, i.e.,

$$
|\langle 5 P\|e r\| 5 D\rangle|=\left(2.069 \pm 0.002_{\text {stat }} \pm 0.04_{\text {syst }}\right) e a_{0} .
$$

In addition, we have become aware of several typographical errors in the paper, which are as follows.

(1) In the caption for Fig. 5 (also appearing in the text of Sec. IV p. 4) the equation should read

$$
\alpha / 2 \pi=(46.57 \pm 0.04) \text {. }
$$

Consequently, the equation at the bottom of the left-hand column of p. 4 should read

$$
\left|\left\langle g\left|e r_{+1}\right| e\right\rangle\right|=\left(0.7167 \pm 0.0006_{\text {stat }} \pm 0.01_{\text {syst }}\right) e a_{0} .
$$

(2) On p. 4 Sec. IV A the equation for the reduced matrix element should read

$$
\left|\left\langle J, m_{J}\left|e r_{+1}\right| J^{\prime}, m_{J^{\prime}}\right\rangle\right|=\left(\begin{array}{ccc}
J & 1 & J^{\prime} \\
-m_{J} & -1 & m_{J^{\prime}}
\end{array}\right)\left|\left\langle J\|e r\| J^{\prime}\right\rangle\right| .
$$

(3) In Table I the correction for vapor cell transmittance should appear as $8.2 \%$. 